\title{
Provisioning in transport networks using power-aware resource allocation algorithms
}

\author{
MADANAGOPAL RAMACHANDRAN* and KRISHNA M SIVALINGAM \\ Department of Computer Science and Engineering, Indian Institute of Technology Madras, Chennai, India \\ e-mail: cs14d009@smail.iitm.ac.in
}

MS received 10 February 2019; revised 3 July 2019; accepted 9 July 2019

\begin{abstract}
Transport technologies such as Optical Transport Network and Synchronous Digital Hierarchy are widely used in access and core networks to carry different types of traffic. These technologies define a hierarchy for multiplexing lower rate traffic containers onto higher rate traffic containers. Provisioning refers to the process of allocation of resources to meet a given traffic demand. Due to the continued expansion of transport networks, the power consumption increases, becoming a bottleneck for further expansion. Power-aware allocation of resources to traffic demands will enable power-efficient operation and help in minimizing the capital and operational costs of the network. In this paper, the power consumed by a switch matrix having the capability to switch different traffic containers at different amounts is considered. The objective is to minimize the number of network elements deployed in the network by allocating resources to traffic demands in power-efficient ways. A formulation based on Integer Linear Programming is first presented; later, four different heuristic approaches, based on how higher order trails are used and the use of grooming, are proposed. It is found that the groomingbased heuristics perform better in terms of the total power consumption relative to the weighted number of requests accepted. In the grooming heuristics, threshold on link utilization is set to determine when grooming is to be done, and for the same performance evaluation, it is found that the threshold of $80 \%$ gives better results.
\end{abstract}

Keywords. Transport networks; power-efficient provisioning; traffic grooming.

\section{Introduction}

With the relentless and significant growth of the Internet, the core and access networks have been expanding continuously. Hence, the capital cost involved in the deployment of network elements to meet the increasing traffic demands is rising. In addition, the power consumption of the network is also increasing due to this expansion. This will eventually become a bottleneck for further expansion since the operational cost is also rising continuously. This mandates the requirement for strategies that take the power consumption of a network into account in order to minimize the capital and operational costs of a network.

Optical Transport Network (OTN) is the de-facto technology used to carry the traffic in national long distance and metro core networks [1]. The Synchronous Digital Hierarchy (SDH) is also a widely used technology to carry different types of traffic in aggregate and access networks [2]. In some countries (e.g. India), around $80-90 \%$ of the transport network elements use SDH technology. Wavelength Division Multiplexing (WDM) is used to carry traffic in metro locations and to carry traffic between locations separated by long distance. If we consider OTN

*For correspondence

Published online: 20 August 2019 over WDM and SDH over WDM, they would account for most of the long distance and metro network traffic requirements in a service provider environment.

OTN and SDH are based on Time Division Multiplexing (TDM). OTN and SDH define a hierarchy for multiplexing lower rate traffic containers into higher rate traffic containers. The multiplexing hierarchy for OTN is defined in [1], where signals at SDH, Ethernet, Fibre Channel, etc. rates are multiplexed onto high rate signals for transmission. The multiplexing hierarchy for SDH is defined in [2], where the low rate G.703 signals such as E1, E4, DS1 and DS3 are multiplexed onto high rate signals for transmission.

Synchronous Optical Networking (SONET) is another technology similar to SDH and widely used in the U.S. and Canada. Its multiplexing structure is similar to that of SDH but with different terminologies: Virtual Tributaries (VT) in SONET instead of Virtual Containers (VC) in SDH. SONET also defines protection mechanisms similar to SDH. This paper focuses on the OTN and SDH standards, but the work can be easily extended to apply to SONETbased networks too.

A typical OTN or SDH network element consists of several line cards that contain ports taking (sending) traffic from (to) different sources (destinations) and a switch 
matrix that switches the traffic among the line cards. Some of the traffic is added or dropped while the rest are passed through. Switch matrix is one or more cards (possibly with backup), which store the cross-connections in circuit-switched transport networks. Switch matrices come with a configured switching capacity, which cannot be exceeded.

The term provisioning refers to the process of allocation of resources to meet a given traffic demand. The focus of this paper is on the design of algorithms for allocating resources to traffic demands in power-efficient ways in transport networks. This is a practical problem given that OTN-over-WDM and SDH-over-WDM are widely used as the underlying transport network by many service providers and also since power is a premium resource in such a network. This problem and the proposed approach is an extension of our earlier work published in COMSNETS 2017 [3], which focussed on a similar approach for SDHover-WDM networks. The problem formulation proposed in [3] has been generalized to support any general transport technology that defines a multiplexing hierarchy such as OTN.

As specified in [3], the amount of traffic between a given source and destination is often the input to a resource allocation algorithm that finds an efficient way of allocating resources that satisfy the given demand. This determines the amount of traffic that is added/dropped at a location or a site. This in turn determines the number of ports of different rates that is required at a location or a site. The switch matrix plays an important role in the power consumed at a location or a site since the amount of traffic that needs to be switched determines the number of network elements required at a location or a site. Since OTN and SDH define different traffic rate containers and a multiplexing hierarchy, the switch matrix specification is in terms of the capability to switch the traffic containers at different amounts.

As specified in [3], since the objective is to minimize the power consumed at a location or a site, the power consumed by a switch matrix and the power consumed by the add/drop tributary OTN or SDH ports are taken as the primary parameters and based on this, an Integer Linear Programming (ILP) model is developed. The model also takes into account the power consumed by the line side ports and amplifiers when the OTN or SDH traffic is carried over a WDM network. Since the problem is NP-Complete, four heuristic algorithms for resource allocation are proposed to handle large networks.

Since the switch matrix that performs switching at higher order layer (ODU-2 in OTN and VC-4 in SDH) consumes less power than equivalent switch matrix at lower order, grooming of multiple lower order signals is performed to reduce the power consumption. A new heuristic based on end-to-end traffic grooming is proposed in this paper, which performs better when compared with the other three heuristics that we have proposed in [3]. The other change with respect to [3] is that the performance evaluation is carried out for different values of grooming threshold in this paper. This threshold applies to the grooming heuristic (GH1) proposed in [3] and the new grooming heuristic (GH2) proposed in this paper, and it is found that grooming performed at threshold of $80 \%$ and above gives better results in terms of total power consumption. GH1 proposed in [3] performs grooming on a per-link basis and $\mathrm{GH} 2$ proposed in this paper performs grooming on a more endto-end basis.

These algorithms can be used for resource allocation during the initial deployment of the network when the traffic demand patterns between different source and destination pairs are known. As manual or automatic provisioning does not take into account the power efficiency aspect during past provisioning, these algorithms can also be used to reorganize a network when the traffic demands between source and destination pairs are available.

The main contributions of the paper are summarized as follows: (i) an ILP formulation that considers switch matrix power consumption and add/drop tributary ports to minimize the total power consumption in a transport network; (ii) four heuristics for resource allocation based on how higher order traffic containers are used and the type of cross-connects that are created; (iii) performances of the heuristics are evaluated among themselves since to the best of our knowledge, there is no earlier work that considers switch matrix power consumption while allocating resources for demands.

The remainder of this paper is organized as follows. Section 2 gives details regarding related work. Section 3 describes the proposed model for power-efficient resource allocation algorithms for provisioning in OTN and SDH networks, and the problem formulation. Section 4 describes the heuristics proposed for power-efficient resource allocation algorithms for OTN and SDH networks. The performance of the algorithms is analysed in detail in section 5. Section 6 concludes the paper.

\section{Background and related work}

This section presents the relevant background and related work.

\subsection{Transport network element architecture}

A typical transport network element containing tributary cards with tributary ports, switch matrix, line cards with network side ports and WDM multiplexer/demultiplexer (mux/demux), and amplifier cards with amplifier ports, is shown in figure 1 . The ports in the tributary cards interface with other network elements from which traffic is received and sent. The switch matrix performs the function of switching the traffic received between an input port and an output port within a network element. The line card converts the aggregate grey signal output into a coloured 


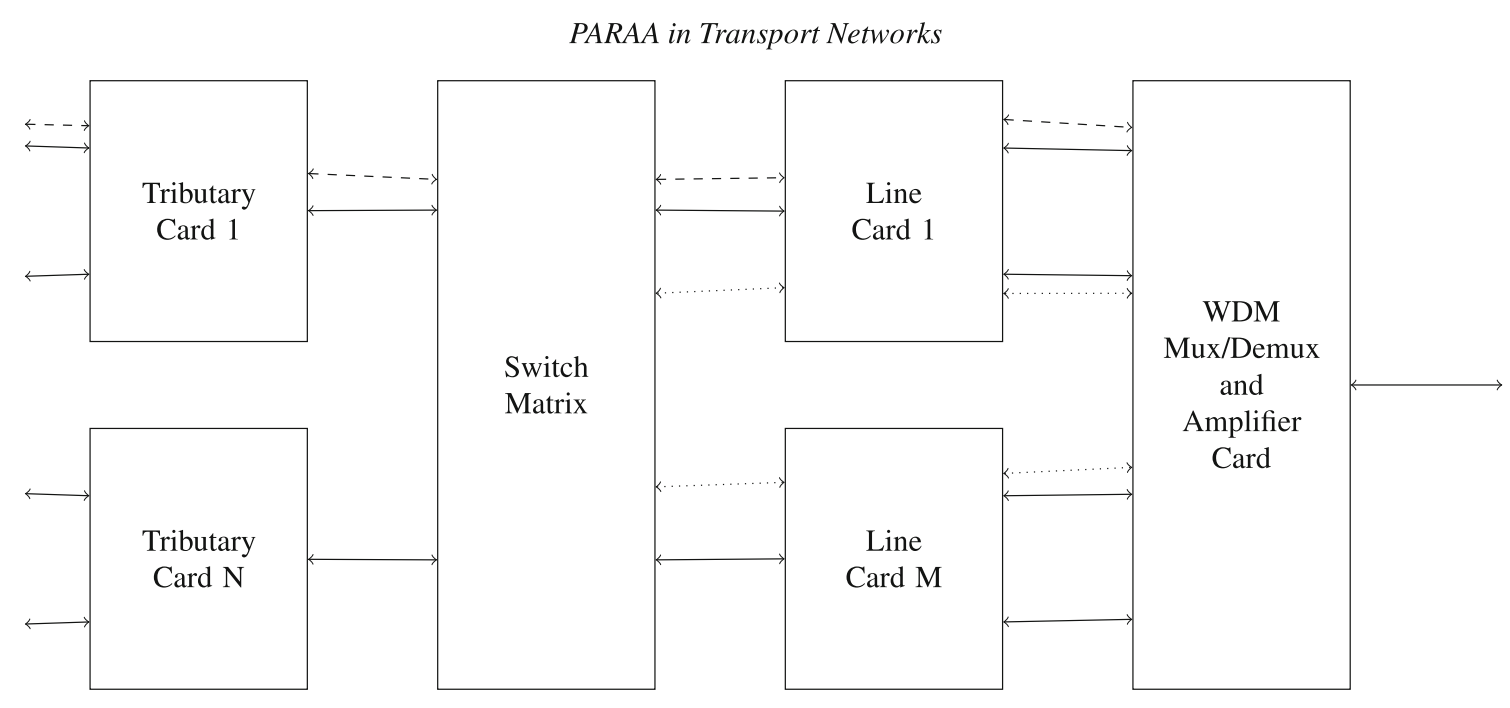

Figure 1. Transport network element.

optical channel that can be transmitted over a WDM segment. The mux/demux cards multiplex or demultiplex many such optical signals. The combined signal is amplified and sent over the optical cable by the amplifier card. This structure is a generalization of the one specified in [3]. The set of dashed lines indicate the traffic added/dropped in Tributary Card 1, which gets switched to Line Card 1 through the switch matrix. The set of dotted lines indicate pass-through traffic from Line Card 1 to Line Card M, which gets switched through the switch matrix.

The OTN operates at bitrates including $100 \mathrm{Gbps}$ (OTU4), 40 Gbps (OTU-3) and 10 Gbps (OTU-2). The SDH synchronous containers operate at bitrates including 155 Mbps (STM-1), 622 Mbps (STM-4), 2.488 Gbps (STM-16), 9.953 Gbps (STM-64), 39.812 Gbps (STM-256) and higher. The corresponding SONET synchronous payload envelope (SPE) capacities are denoted by OC-3, OC-12 and so on.

In the case of OTN, the input SDH, Ethernet, Fibre Channel or other technology traffic is transmitted over an OTN link by the process of mapping the data into different containers. Overhead bytes are then added to the container for operation and management to form optical data units, which are then multiplexed into the final OTN frame that gets transmitted over the OTN link. The different optical data units are denoted as ODU-0 (1.244 Gbps), ODU-1 (2.488 Gbps), ODU-2 (9.953 Gbps) and so on. Of these, ODU- $j(j=2,3,4)$ and above are called higher order rates, and $\mathrm{ODU}_{-j}(j=0,1,2)$ are called lower order rates.

As specified in [3], in the case of $\mathrm{SDH}$, the input Plesiochronous Digital Hierarchy (PDH) or Ethernet or other technology traffic is transmitted over an SDH link by the process of mapping the data into different containers. Overhead bytes are then added to the container for operation and management to form $\mathrm{VC}$, which are then multiplexed into the final SDH frame that gets transmitted over the SDH link. The different VC are denoted as VC-4
(150.336 Mbps), VC-3 (48.96 Mbps), VC-2 (6.848 Mbps), VC-12 (2.24 Mbps) and so on. Of these, VC- $n(n=1,2,3)$ are called lower order VC, and VC- $n(n=3,4)$ are called higher order VC.

As specified in [3], the switching matrix of a transport network element comes with specification on the amount of traffic that it can handle, for different rate traffic containers. For example, a switch matrix could switch $x$ VC-4s and $y$ VC-12s. The power consumed by a larger switch matrix does not increase linearly with the amount of traffic switched by it. For example, the power consumed by a switch that switches $2 x \mathrm{VC}-4 \mathrm{~s}$ and $2 y \mathrm{VC}-12 \mathrm{~s}$ is not twice the power consumed by a switch matrix that switches $x \mathrm{VC}-4 \mathrm{~s}$ and $y$ VC-12s. Similarly, the power consumed by a switch matrix that switches one VC-4 is less than a switch matrix that switches $63 \mathrm{VC}-12 \mathrm{~s}$ even though the amount of traffic switched is equivalent. A similar behaviour is present in OTN switch matrices also.

Power-efficient provisioning in transport networks is different from the model for IP-over-WDM networks in the following ways:

1. Multiple data rates have to be considered in the traffic demand rather than a single data rate.

2. The power consumed by the switch matrix is the main parameter that is minimized.

3. The number of add/drop ports required and their power consumption have to be considered.

\subsection{Path computation algorithms for SDH networks}

Path computation algorithms taking into account the multiplexing hierarchy defined in SDH [2] have been proposed in [4]. The network is treated as a graph containing physical links and logical trails and weights are assigned to them 
before computing a path with the lowest cost. Trails are higher order VC-4 bearers used to multiplex and carry lower order signals like VC-3 and VC-12. Weights are assigned such that the trails are given higher preference to physical links so that existing trails are used wherever possible. In [5], path computation algorithms for dynamic service provisioning with protection and inverse multiplexing in SDH networks are proposed.

\subsection{Energy-minimized design}

A comprehensive survey of research for minimizing energy consumption in telecommunications optical networks is provided in [6]. It describes energy savings mechanisms in core, metro and access networks. In core networks, the various techniques employed are selectively turning off network elements, energy-efficient network design, energyefficient IP packet forwarding and green routing. It also lists the various standardization efforts and also works in data centres and applications.

In [7], an energy-minimized design for IP over WDM networks is proposed, where the number of IP router ports is found to be the major factor that determines the energy consumption and network cost. The energy consumed by the EDFAs and WDM transponders is also taken into account and a Mixed Integer Linear Programming (MILP) formulation is proposed. Since the problem is not tractable for large networks, two heuristics, namely direct bypass and multi-hop bypass, are proposed and it is found that the lightpath bypass strategies result in significant power savings.

Two network architectures namely IP over WDM and IP over OTN over WDM are studied in [8] for energy efficiency and it is shown that CAPEX optimized network results in minimum energy consumption. Also, it is shown that the architecture with the most cost efficiency is not always the one with the most energy efficiency and the network equipment cost is the dominating factor now for cost.

The problem of energy efficiency of mixed line rate (MLR) networks is studied in [9] where the trade-off between capacity and energy consumption is considered. The requirement is to find the optimum number of wavelengths at different data rates to support the given traffic demand and minimize energy consumption. A MILP model is developed for the problem and it is found that a MLR network performs better than a single line rate (SLR) network in terms of energy consumption.

Three heuristic approaches for reducing the power consumption in IP-over-WDM networks by switching off unused line cards are considered in [10]. MILP models using time-varying traffic demands are studied and the proposed heuristic approaches are evaluated and it is shown that most significant energy savings are achieved by monitoring lightpath utilization and switching off empty line cards and rerouting traffic in the IP Layer.
In [11], an approach for considering time-of-day network traffic variation for shutting down idle line cards in IP-overWDM networks is proposed. MILP formulation for modelling the energy consumption of line cards and chassis of IP routers is proposed under static traffic demand in a time period. It is shown that shutting down entire chassis together with line cards results in more energy savings.

The optimal formulation of lifetime-aware network problem is presented in [12], where the link lifetime in IP network is managed through the sleep mode effects. The optimal solution to the problem and a heuristic are presented and it is found that the proposed approach performs better when compared with other approaches, which do not consider lifetime decrease due to power state change.

The network design problem with demand uncertainty is studied in [13], where robust optimization is used for network planning. It is stated that robust optimization, which is used when there is uncertainty in input parameters, can be applicable for integration in network planning tools. Two applications of the problem, namely multi-layer network design and mixed-line-rate network design, are then presented.

\subsection{Traffic grooming}

In [14], power-efficient traffic grooming in optical WDM networks is considered, where the total power consumption in terms of the power consumed by individual lightpaths is modelled. An ILP formulation is developed for the grooming problem where the minimum power consumption is shown to be a generalization of minimization of active router ports and amount of electronically switched traffic. The solution is then shown to result in significant energy savings in small networks.

Two formulations for green optical network design for the traffic grooming problem are provided in [15]. The formulations are either flow-based formulation or interface based, where the three possibilities of how a traffic demand that is not sourced from and destined to a node can be processed. A special case study of a situation that is practical is studied and is shown to result in significant reduction in power consumption.

In [16], energy-efficient traffic grooming in optical networks with sliceable optical transponders is studied where ILP models and heuristics for traffic grooming in three types of bandwidth-variable transponders are proposed. It is found that power savings are achieved using sliceable transponders when the optical transponder sliceability is relatively low.

\section{5 “Green" provisioning}

In [17], the problem of minimizing the operational power in a typical backbone network architecture is considered. An auxiliary graph is proposed that captures the power consumption of each provisioning operation and based on this, a 
power-aware provisioning scheme is developed to minimize the total operational power. It is shown that the scheme requires the least operational power when compared with direct lightpath and traffic grooming approaches.

The problem of virtual optical network provisioning is addressed in [18], where bandwidth requirement on virtual links and computing resources on virtual nodes are used to form primary and backup paths for all node pairs in the physical optical network. Two mapping approaches to reduce power consumption are proposed and it is found that one of the mapping approaches reduces power consumption.

\subsection{Provisioning in multi-layer networks}

In [19], on-demand approach for provisioning in multidomain optical networks is proposed where the utilization of the links is considered. The approach is SDN based where the latency for provisioning is reduced. It maintains the total number of flows as low as possible.

An auxiliary graph-based approach for application-aware service provisioning and restoration in multi-layer transport networks is proposed in [20]. It can be used to dynamically provision applications' service requests and also supports restoration of traffic flows according to their tolerance to service disruption. The proposed approach meets bandwidth, latency and availability requirements and can be extended to consider additional requirements as it is modular.

\subsection{Contributions of this paper}

For resource allocation in transport networks, new ILP model for minimizing the total power consumption and heuristic approaches are required since all the afore-mentioned works do not take into account the multiplexing structure defined in OTN and SDH. Also, power consumed by the switch matrix and the power consumed by add/drop ports have to be considered as the primary parameters that have to be minimized. The main contribution of this paper is the proposal of a new model that takes into the account multiple rate signals defined in OTN and SDH and switch matrix power consumption. Heuristic approaches are then proposed since the problem is NP-Complete. The performances of the proposed heuristic approaches are evaluated among themselves since to the best of our knowledge, there is no earlier work that considers switch matrix power consumption while allocating resources for demands.

\section{Problem formulation}

This section presents the energy-efficient grooming and provisioning problem and formulates it as an ILP problem. The problem is formulated in a generic manner for any multiplexed transport technology such as OTN and SDH.
Problem statement: As specified in [3], the objective is to provision resources to satisfy a given set of traffic demands in a power-efficient manner. The main inputs are the network topology, a traffic demand matrix (explained in detail later) and each switch's traffic carrying capacities.

The provisioning task includes identifying the end-to-end path and allocating the resources to meet a given traffic demand. Allocation of few higher order resources for the input traffic requests and grooming many fine granular or lower order signals will reduce the number of cross-connects in many intermediate nodes. This will result in more power consumption, which can be avoided by grooming the signals appropriately. Existing path computation algorithms such as those proposed in [4] create higher order VC-4 trails between adjacent network elements so that maximum flexibility is achieved in routing traffic demands. From a power efficiency point of view, this is not efficient since this can result in breaking all higher order containers like VC-4s into lower order containers like VC-12s. Another reason is that switching in terms of the lower order containers will consume more power for the reason mentioned in the earlier paragraph. Hence, there is a need to design a new approach for handling traffic demands in a powerefficient manner.

\subsection{ILP formulation}

The provisioning problem is formulated using an ILP model, which is a generalization of the model proposed in [3]. The following are the inputs to the model:

1. A graph of the network $G(V, E)$ where $V$ is the set of locations where network elements need to be deployed and $E$ is the set of fibre links between the locations.

2. $N$ is the set of rates for the technology (OTN or SDH) considered.

3. A demand matrix $[\lambda]$ that might be based on demand forecasts or obtained from an existing demand pattern followed in a deployed network; $\lambda_{s d}$ is the demand between a location pair $(s, d) ; \lambda_{s d, m}$ is the demand for the number of traffic requests at rate $m$. In case of OTN, $m$ could be ODU-0, ODU-1 or ODU- 2 and in the case of SDH, $m$ could be VC-12, VC-2, VC-3 or VC-4.

Table 1. OTN switch matrix power consumption.

\begin{tabular}{lc}
\hline Switch matrix capacity & Power consumption (W) \\
\hline 10 ODU-2 & 2000 \\
5 ODU-2 & 1200 \\
2 ODU-2 equivalent & 650 \\
1 ODU-2 equivalent & 400 \\
\hline
\end{tabular}


Table 2. SDH switch matrix power consumption.

\begin{tabular}{lc}
\hline Switch matrix capacity & Power consumption (W) \\
\hline 16 VC-4 & 1400 \\
8 VC-4 & 800 \\
4 VC-4 equivalent & 550 \\
2 VC-4 equivalent & 320 \\
1 VC-4 equivalent & 200 \\
\hline
\end{tabular}

4. $B_{k}$ is the amount of traffic that is switched by a switch matrix of type $k$. The values for $k$ represent the switch matrix type. In this work, there are four types of OTN switch matrices considered and listed in table 1 and five types of SDH switch matrices considered and listed in table 2 . The total number of switch matrices is denoted by $u . B_{k, m}$ is the amount of traffic switched by a switch matrix of type $k$ at rate $m$. In case of OTN, $m$ could be ODU-0, ODU- 1 or ODU- 2 and in the case of SDH, $m$ could be VC-12, VC-2, VC-3 or VC-4.

5. $C_{k}$ is the power consumed by a switch matrix of type $k$.

6. $F_{l}$ is the amount of traffic that is carried by a tributary port of type $l$. In case of OTN, there are five types of ports OTU-4, OTU-3, OTU-2, OTU-1 and OTU-0 and in case of SDH there are four types of ports STM-1, STM-4, STM-16 and STM-64. The total number of port types is denoted by $v . F_{l, m}$ is the amount of traffic carried by a port of type $l$ at rate $m$.

7. $H_{l}$ is the power consumed by a tributary port of type $l$.

8. $J_{p}$ is the amount of traffic that is carried by a line side port of type $p . J_{p, m}$ is the amount of traffic carried by a line side port of type $p$ at rate $m$.

9. $K_{p}$ is the power consumed by a line side port of type $p$.

10. $Z$ is the power consumed by an amplifier port.

The following are the other variables used in the model:

1. $A_{i}$ is the amount of traffic added at location $i . A_{i, m}$ is the amount of traffic at rate $m$ added at location $i$.

2. $D_{i}$ is the amount of traffic dropped at location $i . D_{i, m}$, is the amount of traffic at rate $m$ dropped at location $i$.

3. $P_{i}$ is the amount of traffic that passes through location $i . P_{i, m}$ is the amount of traffic at rate $m$ that passes through location $i$.

4. $T_{i}$ is the amount of traffic that is added and dropped at and that passes through location $i . T_{i, m}$ is the amount of traffic at rate $m$ that is added and dropped at and passes through location $i$.

5. $\lambda_{s d}^{i j}$ is the demand between a location pair $(s, d)$ that passes through link $(i, j) ; \lambda_{s d, m}^{i j}$ is the demand for the number of requests at rate $m$ between a location pair $(s, d)$ that passes through link $(i, j)$.

6. $M_{i k}$ is the number of switch matrices of type $k$ used at location $i$.
7. $E_{i}$ is the power consumed by all the switch matrices at location $i$.

8. $L_{i j}$ is the total amount of traffic carried in link $(i, j)$. $L_{i j, m}$ is the total amount of traffic at rate $m$ carried in link $(i, j)$.

9. $R_{i l}$ is the number of tributary ports of type $l$ used at location $i$.

10. $X_{i}$ is the power consumed by all the tributary ports at location $i$.

11. $Q_{i j, p}$ is the number of line side ports of type $p$ used at link $(i, j)$.

12. $Y_{i j}$ is the power consumed by all the line side ports at link $(i, j)$.

13. $W_{i j}$ is the number of in-line amplifiers deployed at link $(i, j)$.

All these defined variables are integers; hence, the problem is of type ILP. The objective function is given as

$$
\min \sum_{i \in V}\left(E_{i}+X_{i}\right)+\sum_{(i, j) \in E} 2\left(Y_{i j}+W_{i j} Z\right)
$$

subject to the following constraints:

$$
\begin{gathered}
\sum_{k=1}^{u} M_{i k} C_{k}=E_{i} \quad \forall i \in V, \\
\sum_{l=1}^{v} R_{i l} H_{l}=X_{i} \quad \forall i \in V, \\
\sum_{p=1}^{v} Q_{i j, p} K_{p}=Y_{i j} \quad \forall(i, j) \in E, \\
\sum_{i \in V} \lambda_{s i, m}=A_{s, m} \quad s \in V \quad m \in N, \\
\sum_{i \in V} \lambda_{i d, m}=D_{d, m} \quad d \in V \quad m \in N, \\
\sum_{j \in V, i \neq j, s, d} \lambda_{s d, m}^{i j}=\sum_{j \in V, i \neq j, s, d} \lambda_{s d, m}^{j i}=P_{i, m}, \\
s, d, i \in V, \quad m \in N, \\
A_{i, m}+D_{i, m}+P_{i, m}=T_{i, m} \quad \forall i \in V \quad m \in N \\
\sum_{j \in V, i \neq j, s, d} \lambda_{s d, m}^{i j}=L_{i j, m} \quad s, d, i \in V \quad m \in N, \\
T_{i, m} \leq \sum_{k=1}^{u} M_{i k} B_{k, m} \quad \forall i \in V \quad m \in N, \\
L_{i j, m} \leq \sum_{p=1}^{v} Q_{i j, p} J_{p, m} \quad \forall i, j \in V \quad m \in N . \\
D_{i, m} \leq \sum_{l=1}^{v} R_{i l} F_{l, m} \quad \forall i \in V \quad m \in N, \\
\end{gathered}
$$


The objective function (1) aims to minimize the total power consumed by the complete network consisting of all the locations where network elements will be deployed and the fibre links that carry traffic between the locations. The total power consumed at a location is the sum of the power consumed by all the switch matrices and the tributary add/drop ports deployed in that location. The variables $E_{i}$ and $X_{i}$ denote the power consumed by all the switch matrices and tributary add/drop ports at a location, respectively, and in the first part of the objective function, summation of those variables across all locations is performed. The total power consumed in a link is twice the sum of the power consumed by all the transponder and amplifier ports that form the ends of the link indicated by the variables $Y_{i j}$ and $Z$, respectively, and in the second part of the objective function, summation of those variables across all links is performed.

The constraint for switch matrix power consumption at a location is specified in constraint (2), which is indicated as a product of the number of switch matrices $\left(M_{i k}\right)$ of type $k$ and the power consumed by a switch matrix $\left(C_{k}\right)$ of type $k$. The summation is across all locations $i$. The constraint for tributary add/drop ports at a location is specified in constraint (3), which is indicated as a product of the number of tributary ports $\left(R_{i l}\right)$ of type $l$ and the power consumed by a tributary port $\left(H_{l}\right)$ of type $l$. The summation is across all locations $i$. The constraint for transponder line side ports at a location part of a link is specified in constraint (4), which is indicated as a product of the number of line ports $\left(Q_{i j, p}\right)$ of type $p$ and the power consumed by a line port $\left(K_{p}\right)$ of type $p$. The summation is across all links $(i, j)$.

Constraint (5) specifies the amount of traffic added at a location $i$ indicated by the variable $A_{s, m}$. It is the sum of all the demands for which the source is the location $i$. Constraint (6) specifies the amount of traffic dropped at a location $i$ indicated by the variable $D_{d, m}$. It is the sum of all the demands for which the destination is the location $i$. Constraint (7) specifies the amount of traffic that passes through a location $i$ without getting added or dropped indicated by the variable $P_{i, m}$. It is the sum of all the demands in all the links at a location $i$ for which it is not the source or destination. Constraint (8) specifies the total of the traffic that is added and dropped at and passes through a location $i$ indicated by the variable $T_{i, m}$. The traffic is specified in terms of different rates, which is indicated by the variable $m$.

Constraint (9) specifies the amount of traffic carried in a link $(i, j)$ indicated by the variable $L_{i j, m}$. Constraint (10) specifies that the total traffic of a particular rate that is added, dropped and passes through should be less than the sum of the traffic switched by all the switch matrices that switch traffic in that particular rate. The variable $B_{k, m}$ indicates the amount of traffic switched by a switch matrix of type $k$. Constraint (11) specifies that the total traffic of a particular rate that is added and dropped should be less than the sum of the traffic carried by all the SDH ports that carry traffic at that particular rate. The variable $F_{l, m}$ indicates the amount of traffic carried by a port of type $l$. Constraint (12) specifies that the total traffic of a particular rate that passes through a link should be less than the sum of all the traffic carried by all the transponder ports that carry traffic at that particular rate. The variable $J_{p, m}$ indicates the amount of traffic carried by a line side port of type $p$. The traffic is specified in terms of different rates, which is indicated by the variable $m$.

Since the formulation is based on ILP, which is known to be NP-Complete [21], effective heuristics are required to solve the problem. Also, even for small networks and demand sizes where linear programming solvers could be used, the problem could be infeasible since all the service requests may not be satisfied. If the service requests are such that the ILP becomes infeasible, linear programming solvers give no result. However, a result is required such that most of the service requests are satisfied and a few of them could be rejected. Hence, heuristic approaches for resource allocation are required and the proposed heuristics are presented in the next section.

\section{Heuristic approaches for resource allocation}

Four heuristic approaches are proposed, based on where higher order trails are created and on the type of crossconnect created. Three of them are the same heuristic approaches proposed in [3] and the fourth heuristic is a new approach proposed in this paper.

\subsection{General structure of heuristic algorithm}

The general structure of the algorithm for all the approaches is as follows:

1. Order the traffic demands from the greatest to the lowest.

2. Take the demands one by one and compute the shortest available path that can satisfy that particular demand using the algorithms proposed in [4].

3. Route the traffic demand over the computed path and update the added traffic at the source node, dropped traffic at the destination node and the pass-through traffic at all the intermediate nodes.

4. Once all the demands are routed, the total traffic that is added and dropped at and passed through a node is computed for each location.

5. For each location, the number of switch matrices required to satisfy the total traffic is found by fitting the traffic into the suitable switch matrix type (in terms of capacity) that can satisfy a portion of the total traffic. For example, in case of SDH, if we have switch matrices of type that can switch $100 \mathrm{VC}-12 \mathrm{~s}$, 
$50 \mathrm{VC}-12 \mathrm{~s}$ and $10 \mathrm{VC}-12 \mathrm{~s}$ and we have a total traffic of $380 \mathrm{VC}-12 \mathrm{~s}$, then 3,1 and 3 of those that switch 100,50 and $10 \mathrm{VC}-12 \mathrm{~s}$, respectively, are used. A similar case is applicable for OTN also.

6. For each location, the number of tributary ports required to carry the add/drop traffic is found by fitting the traffic into the suitable port type (in terms of capacity) that can carry a portion of the total add/drop traffic. For example, in case of SDH, if the add/drop traffic is $1800 \mathrm{VC}-12 \mathrm{~s}$, then $1 \mathrm{STM}-16$ port (can carry $1008 \mathrm{VC}-12 \mathrm{~s}$ ), 3 STM-4 ports (each can carry $252 \mathrm{VC}$ $12 \mathrm{~s}$ ) and 1 STM-1 port (can carry $63 \mathrm{VC}-12 \mathrm{~s}$ ) are used. A similar case is applicable for OTN also.

7. For each link, the number of line side ports required to carry the traffic is found by fitting the traffic into the suitable port type (in terms of capacity) that can carry a portion of the total traffic in that link. For example, in case of SDH, if the total link traffic is $1800 \mathrm{VC}-12 \mathrm{~s}$, then $1 \mathrm{STM}-16$ port (can carry 1008 VC-12s), 3 STM-4 ports (each can carry 252 VC$12 \mathrm{~s}$ ) and 1 STM-1 port (can carry $63 \mathrm{VC}-12 \mathrm{~s}$ ) are used. A similar case is applicable for OTN also.

8. The power consumed by all the switch matrices, tributary ports, line side ports and amplifier ports found is then computed.

Computational complexity: The computational complexity for the heuristic algorithm is

$\Theta(\mathscr{D} \log \mathscr{D}+\mathscr{D}((|E|+|V|) \log |V|))$ where $\mathscr{D}$ is the number of demands, $|V|$ is the number of network nodes and $|E|$ is the number of edges. The complexity of the first step is $\Theta(\mathscr{D} \log \mathscr{D})$ for sorting the $\mathscr{D}$ demands. The complexity of the Dijkstra's shortest path algorithm is $\Theta((|E|+|V|)$ $\log |V|)$, which is used to find the path for each demand in $\mathscr{D}$. Therefore, the complexity of the second step is

$\Theta(\mathscr{D}((|E|+|V|) \log |V|))$ for computing the shortest path for each demand in $\mathscr{D}$. The complexity of the third step, which calculates the traffic added or dropped or passes through, is $\Theta(\mathscr{D}|V|)$ since for each demand in $\mathscr{D}$, the path could contain $|V|$ nodes. Steps 4-8 compute the number of switch matrices, ports and total power consumption required to satisfy the given traffic demand, whose complexity is $\Theta(1)$ for each node. Hence, the complexity of the heuristic algorithm is $\Theta((\mathscr{D} \log \mathscr{D})+\mathscr{D}((|E|+|V|) \log |V|))$.

The four heuristics differ in the way higher order trails are created and the type of cross-connect that is created. This is done during the path computation and routing step in the general structure of the algorithm described earlier. The four heuristics are described in sections 4.2-4.5.

\subsection{Adjacent NE trail creation heuristic (ANTCH)}

As specified in [3], this heuristic creates a higher order trail between adjacent locations whenever a new lower order traffic demand has to be routed that cannot be routed over

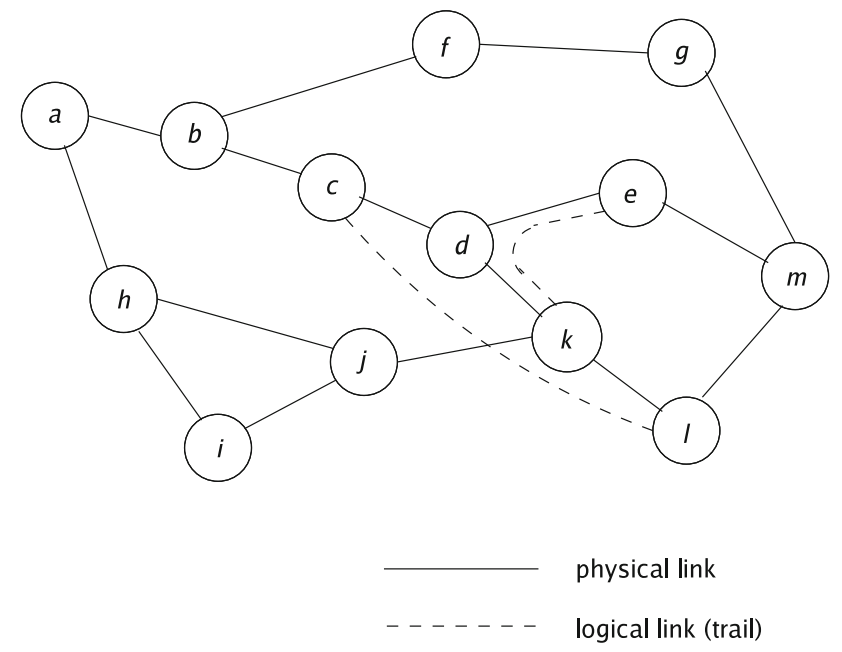

Figure 2. A sample network.

existing higher order trail. This heuristic is chosen since it provides flexibility in routing a lower order traffic demand from any source to any destination as long as there is a path in the network. In terms of switch matrix utilization, one lower order container like ODU-0 (OTN) or VC-12 (SDH) is added to the total at all the locations through which the demand is routed. For example, in the network shown in figure 2 , to route a service request from $b$ to $l$, higher order trails are created between $b$ to $c, c$ to $d, d$ to $k$ and $k$ to $l$. In terms of switch matrix utilization, one cross-connect is created at the locations $b, c, d, k$ and $l$. This means that the count of the lower order containers is added by 1 at that locations.

\subsection{Existing trail usage heuristic (ETUH)}

As specified in [3], this heuristic creates a higher order trail between those locations whenever a new lower order traffic demand has to be routed that cannot be routed over existing higher order trail. This heuristic is chosen since it eases the operations by following the normal way of creating a higher order trail for provisioning a lower order traffic demand. In terms of switch matrix utilization, one lower order container like ODU-0 (OTN) or VC-12 (SDH) is added to the total at the terminating locations of the higher order trails created or used and one higher order container like ODU-2 (OTN) or VC-4 (SDH) is added to the total at all the intermediate locations through which the higher order trail is created. For example, in the network shown in figure 2, to route a service request from $b$ to $l$, higher order trail is created between $b$ and $c$ and the higher order trail between $c$ and $l$ is used. In terms of switch matrix utilization, one lower order cross-connect is created at the locations $b$, $c$ and $l$, and higher order cross-connect is created at the locations $d$ and $k$. This means that the count of the lower order containers is added by 1 at those locations where 
lower order cross-connects are created and the count of the higher order containers is added by 1 at those locations where higher order cross-connects are created.

\subsection{Grooming heuristic 1 (GH1)}

As specified in [3], this heuristic is based on traffic grooming, where grooming is performed on the lower rate container traffic into higher rate containers and switching performed on the higher rate containers. This heuristic is chosen since it provides a tradeoff between flexibility and ease of operations while provisioning a lower order demand over a higher order trail. In terms of switch matrix utilization, whenever a higher order container like ODU-2 (OTN) or VC-4 (SDH) reaches a threshold of full capacity between consecutive links, the equivalent lower order container count is decremented and one ODU-2 (OTN) or $\mathrm{VC}-4(\mathrm{SDH})$ is added to the total at the involving location. For example, in the case of SDH, if $63 \mathrm{VC}-12 \mathrm{~s}$ are being used in consecutive links, VC-12 count is decremented by 63 and VC-4 count is added by 1 at the involving location. In the network shown in figure 2, if the number of crossconnects between a container in the link $b c$ to a container in the link $c d$ reaches a threshold of full capacity, the count of lower order containers like VC-12 is decremented by 63 and the count of the higher order container like $\mathrm{VC}-4$ is incremented by 1 . The threshold percentage is varied from $60 \%$ to $100 \%$ for performance evaluation. A similar case is applicable for OTN also.

\subsection{Grooming heuristic 2 (GH2)}

This heuristic is also based on traffic grooming that performs end-to-end grooming, unlike GH1, which tries to groom within a node for every pair of consecutive links. In this heuristic, after computing the shortest path between the source and destination nodes for all demands, every pair of nodes is processed starting with the pair having the greatest path cost. If path costs are the same, then the pair with greater number of demands between them is processed first. For the current pair of nodes, many end-to-end higher order tunnels (ODU-2 for OTN and VC-4 for SDH) are created between the nodes such that either the tunnel capacity is full or the tunnel usage exceeds the threshold, which is varied from $60 \%$ to $100 \%$ for performance evaluation. Since the tunnels would now carry the demands, demands are assigned to the created tunnels and the demand-related entry is removed from all the nodes part of the tunnel and switch matrix utilization is incremented by the number of higher order tunnels that pass through the nodes in that path of the tunnel. Tunnels are not created for pair of nodes that are one hop away. Using this method, one demand could get carried over multiple tunnels between different pairs of nodes along the route of the demand. After all the node pairs are processed, the total power consumption based on the added, dropped and pass-through traffic and switch matrix utilization are computed.

\subsection{Extension to support protection and inverse multiplexing}

As specified in [3], the proposed heuristic approaches can be used for satisfying traffic demands with dedicated protection involved by using the algorithm for dedicated protection proposed in [5] during the path computation step. Similarly, the algorithm proposed in [5] can be used for satisfying traffic demands with inverse multiplexing rates involved during the path computation step.

\section{Performance results}

The performances of the described heuristics are evaluated and the results obtained are provided in this section. Since there is no earlier work to the best of our knowledge that considers switch matrix power consumption while allocating resources for demands, the performances of the heuristics are evaluated among themselves. The experiments were performed on three following networks: a network with 70 nodes and 103 links (Network 1) [22], a network with 13 nodes and 17 links (Network 2) [23] and on the NSF network with 14 nodes and 21 links (Network 3).

The service requests are randomly generated and the performances of the heuristics are evaluated by running 10 iterations, each with different sets of service requests for Network 1 and Network 2, which are simulated. All the codes for request generation and the heuristic approaches were implemented in Java and the tests were performed on a machine with 4-core Intel i5 processor $(3 \mathrm{GHz}$ ) and 64 GB RAM. In each iteration, service requests are generated as follows:

1. For each iteration, some number of requests are generated such that a small fraction of them are rejected due to resource unavailability. The number of requests generated is 500 for OTN and 1500 for SDH in Network 1 and is 50 for OTN and 200 for SDH in Network 2.

2. Source node and destination node are chosen randomly for each service request.

3. The bandwidth requirement is generated in the following proportion for OTN.

- Distribution 1: ODU-2 - 5\%, ODU-1 - 15\%, ODU$0-80 \%$.

- Distribution 2: ODU-2 - 30\%, ODU-1 - 30\%, ODU-0 - 40\%.

- Distribution 3: ODU-2 - 10\%, ODU-1 - 20\%, ODU-0 - 70\%. 
- Distribution 4: ODU-2 - 10\%, ODU-1 - 30\%, ODU-0 - 60\%.

4. The bandwidth requirement is generated in the following proportion for SDH.

- Distribution 1: VC-4 - 4\%, VC-3 - 10\%, VC-2 $6 \%, \mathrm{VC}-12-80 \%$.

- Distribution 2: VC-4 - 25\%, VC-3 - 25\%, VC-2 $25 \%, \mathrm{VC}-12-25 \%$.

- Distribution 3: VC-4 - 5\%, VC-3 - 10\%, VC-12 $85 \%$.

- Distribution 4: VC-4 - 5\%, VC-3 - 25\%, VC-12 $70 \%$.

The number of requests accepted is an important factor that has to be evaluated. However, since different service requests are for different bandwidth rates, they cannot be treated equally. For example, in case of SDH, a VC-4 is equivalent to $63 \mathrm{VC}-12 \mathrm{~s}$. Hence, an execution that rejects a VC-4 request due to lack of capacity and accepts less than 63 future $\mathrm{VC}-12$ requests will show better performance when compared with an execution that accepts the VC-4 request and rejects the future $\mathrm{VC}-12$ requests. To overcome this, the requests that are accepted have to be weighted according to the relative bandwidth to get a better indication of the performance. This is achieved by keeping VC-12 as the base and multiplying each VC- 4 request by 63 , VC-3 request by 21 and VC-2 request by 3 . In case of OTN, ODU- 0 is kept as the base and ODU- 1 and ODU-2 requests are multiplied by 2 and 8 , respectively. This is referred to as the weighted number of requests accepted.

The power consumption for satisfying all the accepted requests is the other factor that is evaluated. The power consumed by the switch matrices at each location needs to be considered (tables 3 and 4). For this purpose, the switch matrix types specified in tables 1 and 2 are used. These values are arrived at by extrapolating the power

Table 3. OTN port power consumption.

\begin{tabular}{lc}
\hline Port rate & Power consumption (W) \\
\hline OTU-4 & 10000 \\
OTU-3 & 6000 \\
OTU-2 & 1800 \\
OTU-1 & 560 \\
OTU-0 & 360 \\
\hline
\end{tabular}

Table 4. SDH port power consumption.

\begin{tabular}{lc}
\hline Port rate & Power consumption (W) \\
\hline STM64 & 6400 \\
STM16 & 2000 \\
STM4 & 600 \\
STM1 & 180 \\
\hline
\end{tabular}

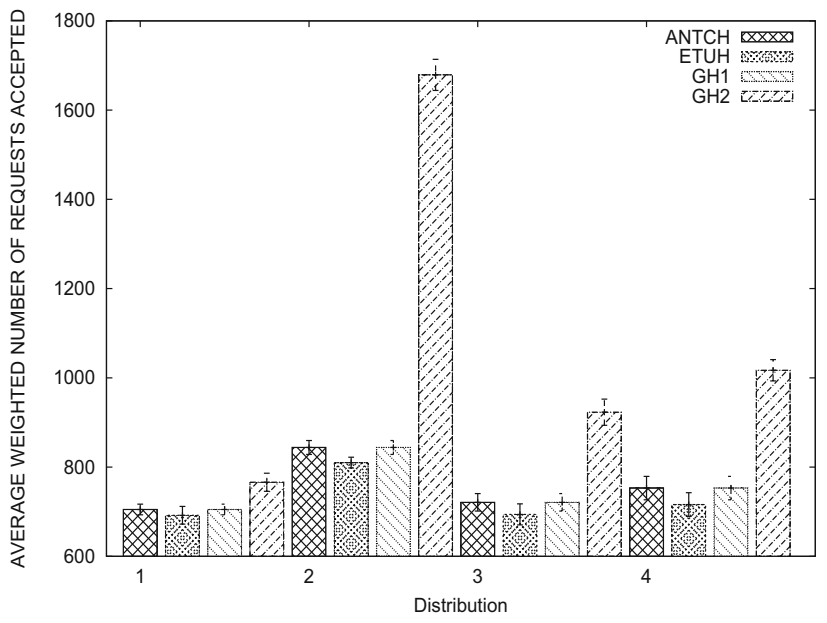

Figure 3. Weighted number of requests accepted for Network 1 with OTN.

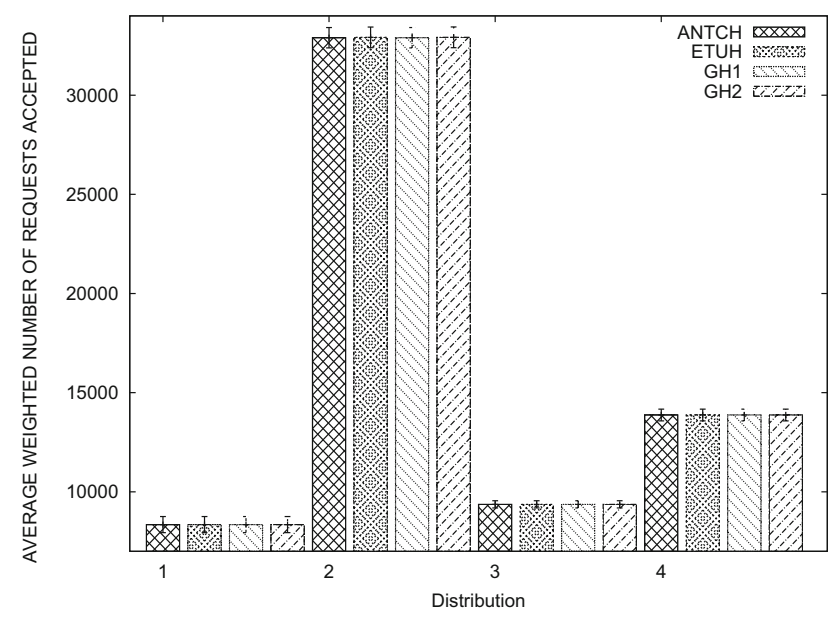

Figure 4. Total power consumption for Network 1 with OTN.

consumption of a typical transport network element that can switch a specified number of traffic containers. Since these network elements switch large amount of traffic containers used in large networks and the performance evaluation is done for small networks in this work, extrapolation is necessary. The power consumed by the tributary and line side ports are specified in tables 3 and 4 .

Since the algorithms proposed in [4] are used in the proposed heuristic approaches, the value of $\alpha$, the relative weight for a higher order trail, needs to be set carefully. This parameter is set as 0.7 since this value is found to give better results when compared with its lower and higher values. The experiments are run for Network 1 and Network 2, 10 times each with different sets of random service requests. The average of the results obtained in the 10 iterations for the two factors mentioned earlier with a confidence interval of $95 \%$ is shown in figures 3 and 4 for OTN in Network 1 and in figures 5 and 6 for SDH in Network 1. 


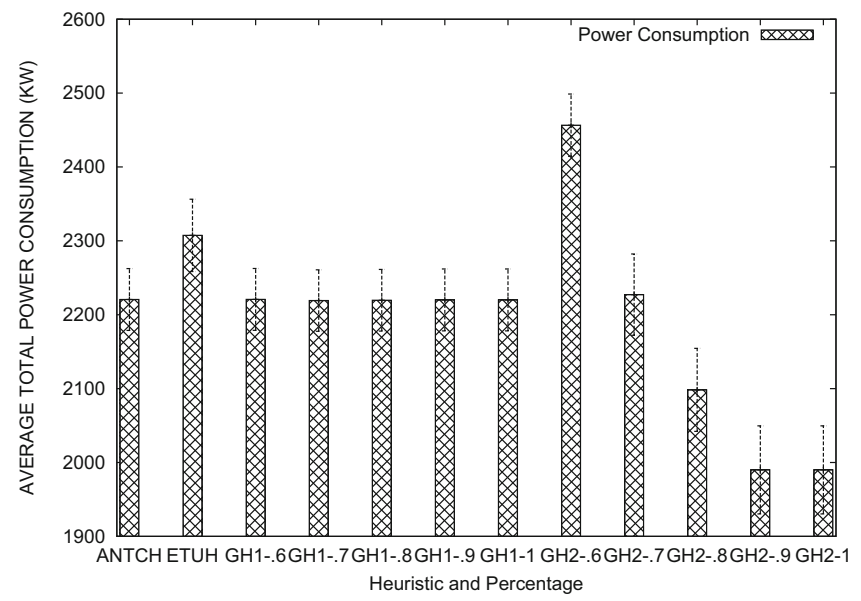

(a) Distribution 1

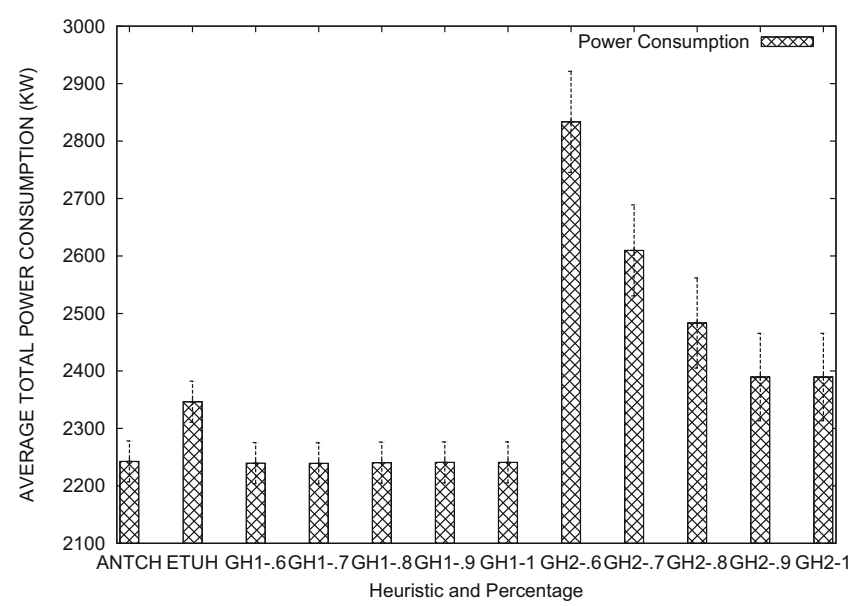

(c) Distribution 3

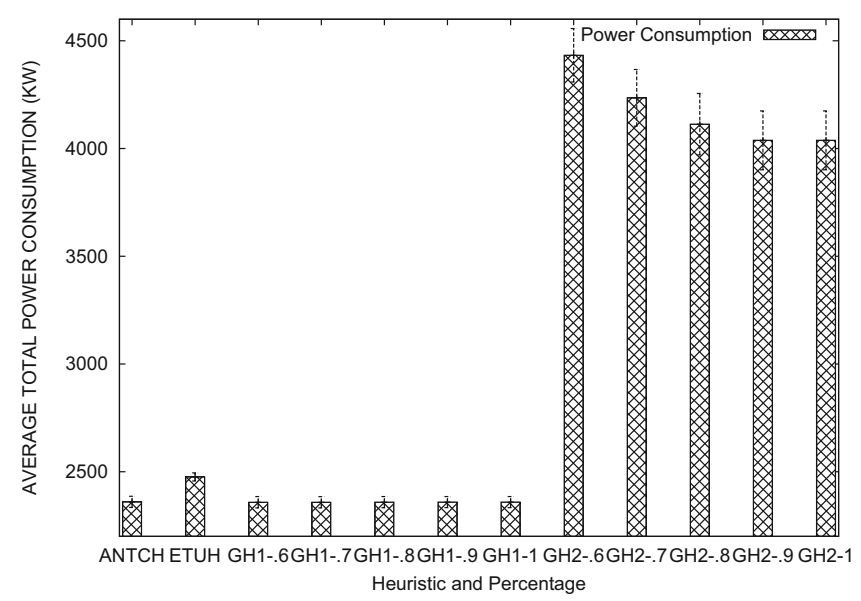

(b) Distribution 2

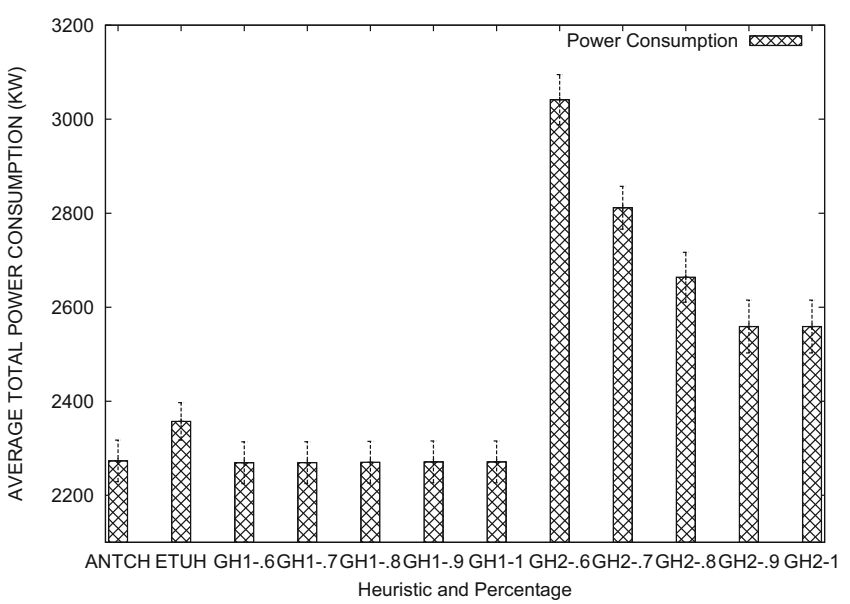

(d) Distribution 4

Figure 5. Weighted number of requests accepted for Network 1 with SDH.

From the results, it is observed that the weighted number of requests accepted is the same for $\mathrm{ANTCH}, \mathrm{GH} 1$ and $\mathrm{GH} 2$ and it is greater than that for ETUH. The total power consumption for ETUH is generally higher than that for ANTCH, GH1 and GH2 but in some cases it is less than that for ANTCH, GH1 and GH2. This is observed only in $\mathrm{SDH}$ case, where the weighted number of requests accepted is somewhat less for ETUH compared with the other two heuristics. This in turn resulted in less power consumption for ETUH. For Network 2, the results obtained are similar to those for Network 1 but with less difference due to smaller size of the network and lesser number of requests used.

To obtain the correct inferences from the results obtained, the weighted number of requests accepted relative to the total power consumption has to be the factor considered since this is the right indicator of the performance of the heuristic approaches proposed. This is defined as follows:

$$
\eta_{x, y}=\alpha_{x, y} / \beta_{x, y}
$$

where $\eta_{x, y}$ is the relative factor for the distribution $x$ and heuristic $y, \alpha_{x, y}$ is the weighted number of requests accepted for the distribution $x$ and heuristic $y$ and $\beta_{x, y}$ is the total power consumption for the distribution $x$ and heuristic $y$.

Higher value for this factor means that the approach performs better since the total power consumption will be lesser for more weighted number of requests accepted. This factor is the highest for $\mathrm{GH} 2$ followed by $\mathrm{GH} 1$, which is higher than that for ANTCH, which is in turn higher than that for ETUH as shown in tables 5 and 6 . For GH1, the threshold percentage of $80 \%$ and sometimes $90 \%$ has the highest value for this factor for OTN and percentage of $70 \%$ and sometimes $80 \%$ for SDH. For GH2, the threshold percentage of $100 \%$ has the highest value for this factor followed by $90 \%$ and $80 \%$ for both OTN and SDH.

This implies that GH2 and GH1, which groom traffic, perform better than the other two heuristics and the 


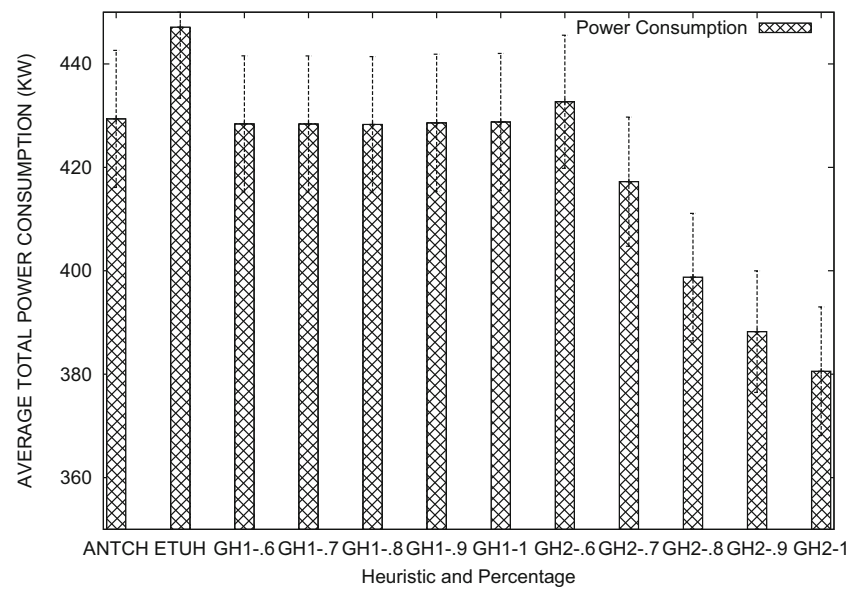

(a) Distribution 1

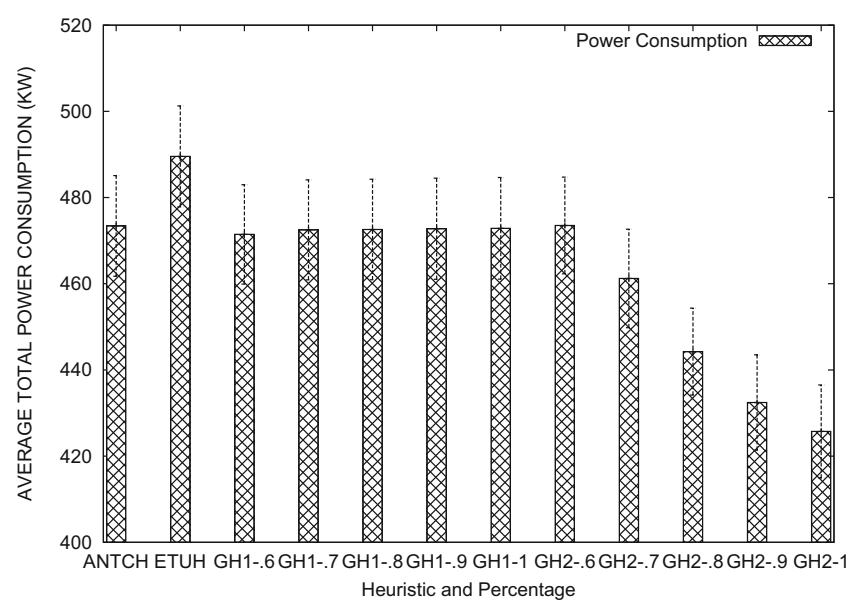

(c) Distribution 3

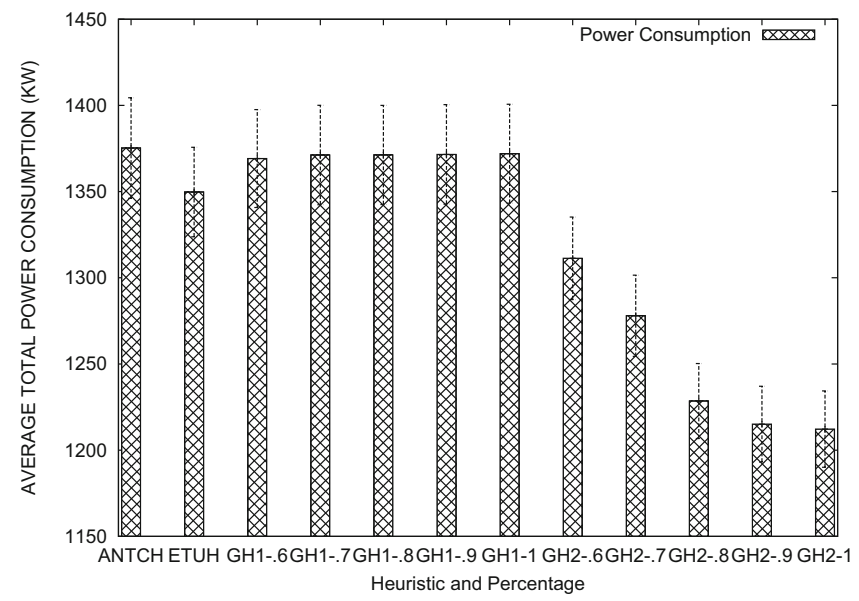

(b) Distribution 2

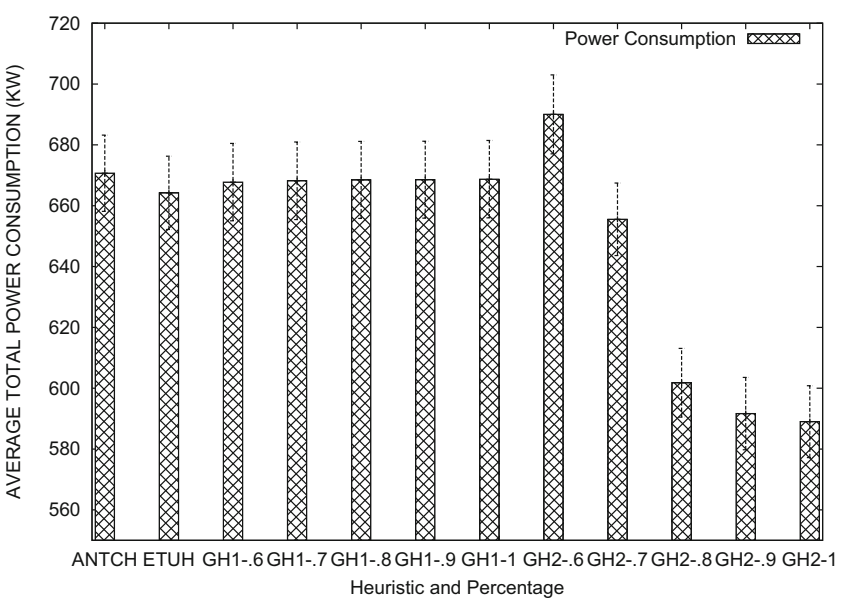

(d) Distribution 4

Figure 6. Total power consumption for Network 1 with SDH.

Table 5. Relative factor $(\eta)$ for Network 1 with OTN.

\begin{tabular}{lcccc}
\hline Heuristic & Dist. 1 & Dist. 2 & Dist. 3 & Dist. 4 \\
\hline ANTCH & .3174 & .3576 & .3215 & .3312 \\
ETUH & .2998 & .3271 & .2957 & .3037 \\
GH1 $(60 \%)$ & .3174 & .3578 & .3219 & .3318 \\
GH1 $(70 \%)$ & .3176 & .3579 & .3219 & .3317 \\
GH1 $(80 \%)$ & .3176 & .3578 & .3218 & .3316 \\
GH1 $(90 \%)$ & .3175 & .3577 & .3217 & .3315 \\
GH1 $(100 \%)$ & .3175 & .3577 & .3217 & .3315 \\
GH2 $(60 \%)$ & .3118 & .3787 & .3257 & .3343 \\
GH2 $(70 \%)$ & .3439 & .3964 & .3536 & .3617 \\
GH2 $(80 \%)$ & .3650 & .4082 & .3716 & .3817 \\
GH2 $(90 \%)$ & .3849 & .4158 & .3863 & .3974 \\
GH2 $(100 \%)$ & .3849 & .4158 & .3863 & .3974 \\
\hline
\end{tabular}

ANTCH performs better than the ETUH. This is because grooming results in higher order cross-connect getting created in the switch matrices for which the power
Table 6. Relative factor $(\eta)$ for Network 1 with SDH.

\begin{tabular}{lcccc}
\hline Heuristic & Dist. 1 & Dist. 2 & Dist. 3 & Dist. 4 \\
\hline ANTCH & .0194 & .0239 & .0197 & .0206 \\
ETUH & .0186 & .0243 & .0191 & .0208 \\
GH1 $(60 \%)$ & .0194 & .0240 & .0198 & .0207 \\
GH1 $(70 \%)$ & .0194 & .0239 & .0198 & .0207 \\
GH1 $(80 \%)$ & .0194 & .0239 & .0198 & .0207 \\
GH1 $(90 \%)$ & .0194 & .0239 & .0198 & .0207 \\
GH1 $(100 \%)$ & .0194 & .0239 & .0198 & .0207 \\
GH2 $(60 \%)$ & .0192 & .0251 & .0197 & .0201 \\
GH2 $(70 \%)$ & .0200 & .0257 & .0203 & .0211 \\
GH2 $(80 \%)$ & .0209 & .0267 & .0210 & .0230 \\
GH2 $(90 \%)$ & .0214 & .0270 & .0216 & .0234 \\
GH2 $(100 \%)$ & .0219 & .0271 & .0220 & .0235 \\
\hline
\end{tabular}

consumption is less compared with the power consumption by equivalent lower order cross-connects created in the switch matrices. Among the two grooming heuristics, GH1 


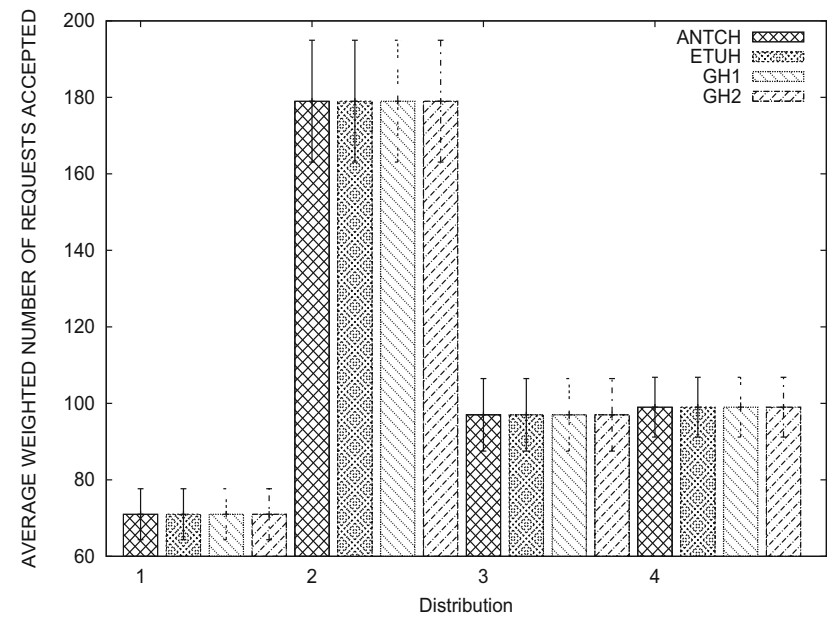

Figure 7. Weighted number of requests accepted in Network 2 for OTN.

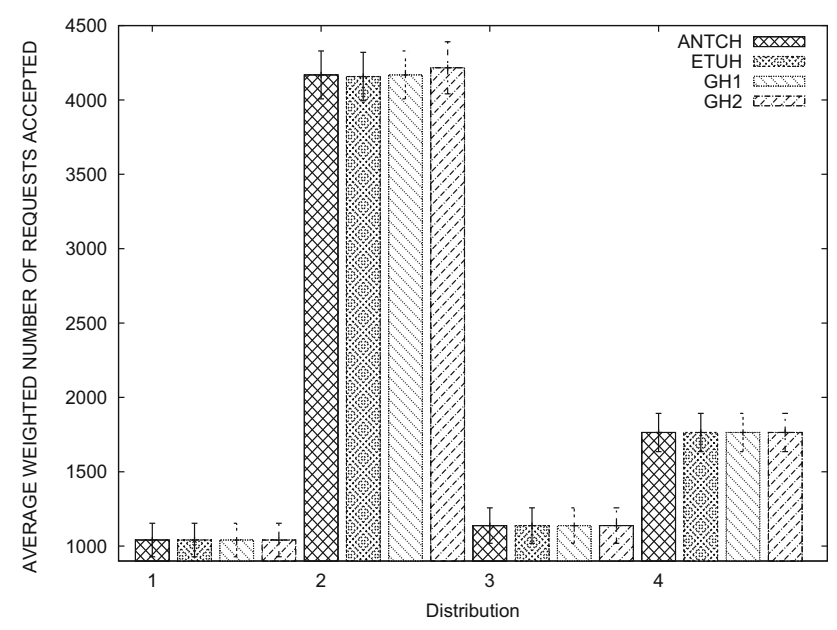

Figure 8. Weighted number of requests accepted in Network 2 for SDH.

Table 7. Relative factor $(\eta)$ for Network 3 with OTN.

\begin{tabular}{lcccc}
\hline Heuristic & Dist. 1 & Dist. 2 & Dist. 3 & Dist. 4 \\
\hline ANTCH & .2620 & .3018 & .2687 & .2764 \\
ETUH & .1337 & .2254 & .1543 & .1660 \\
GH1 $(60 \%)$ & .2611 & .3020 & .2679 & .2759 \\
GH1 $(70 \%)$ & .2617 & .3020 & .2685 & .2761 \\
GH1 $(80 \%)$ & .2619 & .3019 & .2687 & .2765 \\
GH1 $(90 \%)$ & .2620 & .3019 & .2687 & .2765 \\
GH1 $(100 \%)$ & .2620 & .3019 & .2687 & .2765 \\
GH2 $(60 \%)$ & .2625 & .3098 & .2697 & .2750 \\
GH2 $(70 \%)$ & .2859 & .3159 & .2891 & .2937 \\
GH2 $(80 \%)$ & .2962 & .3190 & .2976 & .3063 \\
GH2 $(90 \%)$ & .3028 & .3237 & .3007 & .3151 \\
GH2 $(100 \%)$ & .3028 & .3237 & .3007 & .3151 \\
\hline
\end{tabular}

and $\mathrm{GH} 2$, better performance is observed for $\mathrm{GH} 2$ since it performs end-to-end grooming, which is more optimistic than GH1, which is conservative. The ETUH that uses the
Table 8. Relative factor $(\eta)$ for Network 3 with SDH.

\begin{tabular}{ccccc}
\hline Heuristic & Dist. 1 & Dist. 2 & Dist. 3 & Dist. 4 \\
\hline ANTCH & .0378 & .0483 & .0378 & .0406 \\
ETUH & .0355 & .0481 & .0356 & .0394 \\
GH1 $(60 \%)$ & .0376 & .0483 & .0378 & .0406 \\
GH1 $(70 \%)$ & .0377 & .0483 & .0378 & .0406 \\
GH1 $(80 \%)$ & .0377 & .0483 & .0378 & .0407 \\
GH1 $(90 \%)$ & .0378 & .0483 & .0378 & .0407 \\
GH1 $(100 \%)$ & .0378 & .0483 & .0378 & .0407 \\
GH2 $(60 \%)$ & .0384 & .0503 & .0385 & .0407 \\
GH2 $(70 \%)$ & .0395 & .0509 & .0389 & .0417 \\
GH2 $(80 \%)$ & .0407 & .0525 & .0405 & .0439 \\
GH2 $(90 \%)$ & .0411 & .0530 & .0412 & .0448 \\
GH2 $(100 \%)$ & .0415 & .0531 & .0415 & .0450 \\
\hline
\end{tabular}

trails already created results in the comparatively worst performance since it may use longer routes getting used, which means lesser weighted number of requests accepted and more power consumption. Similar results are obtained for Network 2 also, which is shown in figures 7 and 8 for the average weighted number of requests accepted in OTN and SDH, respectively. Similar results are obtained for average total power consumption and they have not been shown due to lack of space.

In Network 3, 100 and 400 requests are generated for OTN and SDH, respectively, and similar results are obtained as shown in tables 7 and 8, where $\mathrm{GH} 2$ followed by $\mathrm{GH} 1$ gives better results for the relative factor for both OTN and SDH networks.

Comparison to ILP results

The performance of the heuristics is then compared to the optimal solution obtained by solving the formulated problem using an LP solver; lp solve [24] is the software used to solve the ILP problem on a desktop with 2.40 $\mathrm{GHz}$ CPU and 4 GB memory. For Network 2, there were 100 requests generated in the proportion mentioned earlier and the problem solved using the LP solver. The results obtained for the resource allocation and power consumption at each location are identical to the results obtained using GH1 and GH2. This is the case when the problem is feasible (all the service requests can be satisfied). The same result is obtained when Gurobi [25] commercial solver is also used. The time taken for computing the result by Gurobi solver is significantly higher compared with the heuristic approach proposed. For Network 2, when 200 requests are generated, the Gurobi solver took 1 hour to compute the result whereas the heuristic approach proposed took less than a minute; lp_solve took even more time for smaller number of requests.

As specified in [3], when the problem becomes infeasible, $l p$ solve can report only failure for the entire problem whereas the heuristics can be used to get partial results for those service requests that can be satisfied. 
Further, for problems involving large networks, LP solvers cannot be used since the number of variables and constraints will be very high. Since the heuristics give identical results when compared to an LP solver, when combined with the fact that they can be used for large networks to satisfy the service requests fully or partially, they can be effectively used to allocate resources in power-efficient ways.

\section{Conclusions}

In this paper, new resource allocation algorithms for powerefficient service provisioning in transport networks are presented. These algorithms differ from the algorithms proposed for IP-over-WDM networks in that they take into account the power consumed by switch matrices that switch different traffic containers in different amounts. An ILPbased formulation is first presented.

Four heuristic approaches are proposed to solve the problem. The first heuristic does not groom traffic; the second heuristic uses existing trails as much as possible; the third heuristic grooms traffic when there is an opportunity and the fourth heuristic performs end-to-end traffic grooming, which is more optimistic. The performances of the approaches are then evaluated and it is found that the grooming heuristics perform better compared with the other two heuristics in terms of the total power consumption relative to the weighted number of requests accepted. It is also found that the performances of the heuristics are identical to the one obtained by solving the problem using an LP solver.

\section{Acknowledgements}

This work was supported by an IRDA grant from IIT Madras (2017-2020) and by a DST grant (EMR/2016/ 003016) from Government of India (2017-2020).

\section{References}

[1] ITU-T 2016 Interfaces for the optical transport networks (OTN). ITU-T Recommendation G.709

[2] ITU-T 2000 Network node interface for the synchronous digital hierarchy (SDH). ITU-T Recommendation G.707/Y.1322

[3] Ramachandran M and Sivalingam K M 2017 Power efficient resource allocation algorithms for provisioning in SDH networks. In: Proceedings of the 9th International Conference on COmmunication Systems and NetworkS (COMSNETS), pp. 282-289

[4] Madanagopal R, Rani NU and Gonsalves TA 2007 Path computation algorithms for dynamic service provisioning in SDH networks. In: Proceedings of the 10th IFIP/IEEE Symposium on Integrated Management (IM), pp. 206-215
[5] Madanagopal R, Rani NU and Gonsalves TA 2010 Path computation algorithms for dynamic service provisioning with protection and inverse multiplexing in SDH/SONET networks. IEEE/ACM Trans. Netw. 18(5): 1492-1504

[6] Zhang Y, Chowdhury P, Tornatore M and Mukherjee B 2010 Energy efficiency in telecom optical networks. IEEE Commun. Surv. Tutor. 12(4): 441-458

[7] Shen G and Tucker RS 2009 Energy-minimized design for IP over WDM networks. IEEE/OSA J. Opt. Commun. Netw. 1(1): 176-186

[8] Palkopoulou E, Schupke D A and Bauschert T 2009 Energy efficiency and capex minimization for backbone network planning: is there a tradeoff? In: Proceedings of the 3rd IEEE International Symposium on Advanced Networks and Telecommunication Systems (ANTS), pp. 1-3

[9] Chowdhury P, Tornatore M and Mukherjee B 2010 On the energy efficiency of mixed-line-rate networks. In: Proceedings of OFC/NFOEC 2010

[10] Idzikowski F, Orlowski S, Raack C, Woesner H and Wolisz A 2011 Dynamic routing at different layers in IP-over-WDM networks maximizing energy savings. Opt. Switch. Netw. 8(3): 181-200

[11] Zhang Y, Tornatore M, Chowdhury P and Mukherjee B 2011 Energy optimization in IP-over-WDM networks. Opt. Switch. Netw. 8(3): 171-180

[12] Chiaraviglio L, Amorosi L, Dellólmo P, Liu W, Gutierrez JA, Cianfrani A, Polverini M, Le Rouzic E and Listanti M 2017 Lifetime-aware ISP networks: optimal formulation and solutions. IEEE/ACM Trans. Netw. 25(3): 1924-1937

[13] Bauschert T, Busing C, DÁndreagiovanni F, Koster A C, Kutschka M and Steglich U 2014 Network planning under demand uncertainty with robust optimization. IEEE Commun. Mag. 52(2): 178-185

[14] Yetginer E, Rouskas G N 2009 Power efficient traffic grooming in optical WDM networks. In: Proceedings of IEEE GLOBECOM

[15] Huang S, Seshadri D and Dutta R 2009 Traffic grooming: a changing role in green optical networks. In: Proceedings of IEEE GLOBECOM

[16] Zhang J, Zhao Y, Yu X, Zhang J, Song M, Ji Y and Mukherjee B 2015 Energy-efficient traffic grooming in sliceable-transponder-equipped IP-over-elastic optical networks [invited]. J. Opt. Commun. Netw. 7(1): A142A152

[17] Xia M, Tornatore M, Zhang Y, Chowdhury P, Martel C U and Mukherjee B 2011 Green provisioning for optical WDM networks. IEEE J. Sel. Top. Quantum Electron. 17(2): 437-445

[18] Chen B 2016 Power-aware virtual optical network provisioning in flexible bandwidth optical networks [invited]. Photon. Netw. Commun. 32(2): 300-309

[19] Biswas M I, Morrow P, Abu-Tair M, McClean S, Scotney B and Parr G 2018 A SDN-based on-demand path provisioning approach across multi-domain optical networks. In: Proceedings of the 20th IEEE International Conference on High Performance Computing and Communications; 16th IEEE International Conference on Smart City; 4th IEEE International Conference on 
Data Science and Systems (HPCC/SmartCity/DSS), pp. 903-908

[20] Savi M and Siracusa D 2018 Application-aware service provisioning and restoration in SDN-based multi-layer transport networks. Opt. Switch. Netw. 30: 71-84

[21] Schrijver A 1998 Theory of linear and integer programming. West Sussex, England: John Wiley \& Sons
[22] 70 node network diagram. http://www.cse.iitm.ac.in/\% 7Eskrishnam/nwdiagram1.png

[23] 13 node network diagram. http://www.cse.iitm.ac.in/\% 7Eskrishnam/nwdiagram2.png

[24] lp\_solve Reference Guide. http://lpsolve.sourceforge.net/5. $0 /$

[25] Gurobi Optimization. http://www.gurobi.com 